\title{
CirCUlating MiCRORNA-30e PREDICTS LEFT VENTRICULAR HYPERTROPHY IN ESSENTIAL HYPERTENSIVE PATIENTS
}

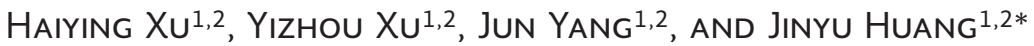 \\ Department of Cardiology, ${ }^{1}$ The Fourth Clinical Medical College, Zhejiang Chinese Medical University, Hangzhou, \\ Zhejiang and ${ }^{2}$ Hangzhou First People's Hospital of Zhejiang University, Hangzhou, Zhejiang, China
}

\begin{abstract}
Background: The biomarker for left ventricular hypertrophy (LVH) in patients with essential hypertension (EH) remains an unmet clinical need. The microRNA-30 (miR-30) family has been associated with LVH in cellular and animal studies, but not in a clinical setting. Objective: The objective of the study was to investigate the clinical significance of circulating levels of miR-30 family as a biomarker for LVH in EH patients. Methods: A total of $239 \mathrm{EH}$ patients and 239 healthy controls were enrolled in this study. Circulating levels of miR-30 family members, namely, miR-30a, miR-30b, miR-30c-1, miR-30c-2, miR$30 \mathrm{~d}$, and miR-30e, were evaluated using real-time polymerase chain reaction assays. Results: The circulating miR-30a, miR-30b, and miR-30e were significantly reduced in EH patients in contrast to controls. EH patients with LVH (EH-LVH) had substantially lower circulating miR-30b and miR-30e levels compared to EH patients without LVH (EH-nLVH). Moreover, the expression levels of miR-30b and miR-30e were positively related to LVMI, respectively. Receiver operating curve analysis showed that circulating miR-30e levels distinguished EH patients from controls, and EH-LVH from EH-nLVH patients. Logistic regression analysis identified the circulating miR-30e as a risk factor for LVH in EH patients. Conclusion: Circulating miR-30e level can be used as a biomarker in distinguishing EH-LVH from EH-nLVH. A further prospective study is warranted to validate this finding. (REV INVEST CLIN. 2021;73(3):XX-XX)
\end{abstract}

Key words: Essential hypertension. Left ventricular hypertrophy. MicroRNA.

\section{INTRODUCTION}

Left ventricular hypertrophy (LVH) is a major complication of essential hypertension $(\mathrm{EH})^{1}$. Approximately one-third of $\mathrm{EH}$ patients develop $\mathrm{LVH}^{2,3}$. The presence of LVH has been identified as a strong independent risk factor for cardiac events and all-cause mortality in the long-term ${ }^{4,5}$. Thus, early detection of LVH is critical for the long-term outcome of EH patients. Although traditional methods, such as electrocardiography, echocardiography, computed tomography, and magnetic resonance imaging, have been widely used to detect LVH, their effectiveness can be hindered by low sensitivity and specificity,
*Corresponding author:

Jinyu Huang

E-mail: jinyuhuangg@hotmail.com
Received for publication: 29-08-2020

Approved for publication: 30-10-2020

DOI: $10.24875 / R I C .20000447$

0034-8376 / (c) 2020 Revista de Investigación Clínica. Published by Permanyer. This is an open access article under the CC BY-NC-ND license (http://creativecommons.org/licenses/by-nc-nd/4.0/). 
lengthy diagnostic time, and technical complexity. Therefore, novel methods for LVH detection with adequate accuracy, easy access, and low cost remain an unmet clinical need.

MicroRNAs (miRNAs) are small ( 22 nucleotides in length), endogenous, non-coding RNAs that degrade the target messenger RNA or by direct translational inhibition, thus blocking the expression of specific genes $^{6}$. The fundamental roles (miRAN) play in diverse biological and pathologic processes, including cell development, differentiation, proliferation, and apoptosis, have been widely studied ${ }^{7}$. Of note, miRNAs are expressed not only in tissues but are also detectable in peripheral blood circulation. The miRNAs levels in circulating blood are not only stable but also they are easy to obtain, making them excellent surveillance approaches for physiological and/or pathological conditions. To date, circulating miRNAs have been used as easily accessible biomarkers to monitor diseases such as cancer radio-sensitivity and metastasis ${ }^{7-9}$.

The previous studies have shown that miRNAs play pivotal roles in the development of cardiac hypertrophy and subsequent cardiac dysfunction ${ }^{10-13}$. Of these miRNAs, the miR-30 family, which is encoded by six genes located on human chromosomes 1,6 , and 8 , is abundantly expressed in cardiomyocytes ${ }^{14,15}$. The miR-30 family contains six members, namely, miR30a, miR-30b, miR-30c-1, miR-30c-2, miR-30d, and miR-30e. These miRNAs share a common seed sequence near the $5^{\prime}$ end. However, they possess different compensatory sequences near the $3^{\prime}$ end, allowing them to target diverse genes and pathways and perform the corresponding biological functions ${ }^{16}$.

It has been reported that the miR-30 family is critical in regulating the expression levels of genes responsible for autophagy, apoptosis, oxidative stress, inflammation, and fibrosis, which are the pathological basis of LVH development ${ }^{17-19}$. Indeed, the role of miR-30 family in cardiomyocyte hypertrophy has been reported in cultured cells and animal studies ${ }^{17,20,21}$; however, the significance of mir-30 family members in a clinical setting has not been evaluated so far. In this study, we measured the circulating levels of the above miR-30 family members in $\mathrm{EH}$ patients. We sought to explore their clinical significance in distinguishing $\mathrm{EH}$ patients with $\mathrm{LVH}(\mathrm{EH}-\mathrm{LVH})$ from those without (EH-nLVH).

\section{METHODS}

\section{Study population}

In this matched case-control study, we recruited $239 \mathrm{EH}$ patients, 90 with LVH (EH-LVH) and 149 without (EH-nLVH), and 239 healthy subjects with normal blood pressure (Controls). Hypertension was diagnosed according to the World Health Organization criteria when presenting a systolic blood pressure (SBP) of $140 \mathrm{mmHg}$ or above, or diastolic blood pressure (DBP) of $90 \mathrm{mmHg}$ or above. All enrolled subjects had no other concomitant diseases, including another form of hypertension in addition to essential hypertension, body mass index (BMI) greater than $35 \mathrm{~kg} / \mathrm{m}^{2}$, cancer, heart valve disease, acute coronary artery disease or acute myocardial infarction, Chagas disease, bundle branch block, and ventricular pre-excitation syndromes.

\section{Ethics}

All procedures followed were in accordance with the Helsinki Declaration of 1975 , as revised in 1983. The institutional Ethics and Clinical Research Committee of the Hangzhou First People's Hospital approved the study (ZDHZ-01A), and all subjects in this study gave written informed consent at enrollment.

\section{Data collection}

Clinical data regarding blood pressure values, diabetes mellitus (DM), and smoking history were collected on admission. BMI was calculated according to each participant's weight and height. Biochemical variables, including serum creatinine, total cholesterol (TC), total triglycerides (TG), high-density lipoprotein cholesterol (HDL-C), and low-density lipoprotein cholesterol (LDLC), were retrieved from the patient's medical chart.

\section{Echocardiographic measurements}

Echocardiographic examinations were performed using a $2.5-\mathrm{MHz}$ probe with a Vivid 7 Pro (GE Vingmed, Horten, Norway) echocardiography device. Echocardiographic imaging was performed according to the recommendations of the American Society of Echocardiography. 12 LVM was calculated according to the Devereux formula. $13 \mathrm{LVM}(\mathrm{gr})=0.8 \times\{1.04 \times$ $[(p W T d+$ IVSd + LVEDD) $3-($ LVEDD $) 3\}+0.6$, where 
d is end - diastole, PWTd is the posterior wall thickness, IVSd is interventricular septal thickness, and LVEDD is LV end - diastolic dimension. Subsequently, relative wall thickness was calculated as the ratio: $2 \times$ (LV posterior wall thickness/LV end - diastolic diameter). LV hypertrophy was defined as increased LVM index (LVM/body surface area) $\geq 115 \mathrm{~g} / \mathrm{m}^{2}$ for men and $\geq 95 \mathrm{~g} / \mathrm{m}^{2}$ for women, as proposed by the European Society of Hypertension ${ }^{22}$.

\section{RNA isolation and reverse transcription and real-time polymerase chain reaction (rt-PCR)}

About $5 \mathrm{~mL}$ venous blood samples were collected in heparin treated tubes from early morning fasting venous blood of all participants. Total RNA was extracted using the RNAprep Pure Hi-Blood Kit (Qiagen Inc). Reverse transcription was conducted using a PrimeScript RT reagent kit (Takara Bio, Japan). Expressions of miR30 family members were performed by quantitative rtqPCR with the SYBR Premix Ex Taq II (Takara, Dalian, China). The rt-qPCR reaction contained $10 \mu \mathrm{L}$ SYBR Premix, $0.8 \mu \mathrm{L}$ forward primer, $0.8 \mu \mathrm{L}$ reverse primer, 2 $\mu \mathrm{L}$ cDNA, and $6.4 \mu \mathrm{L}$ nuclease-free water. All samples were performed in triplicate. Glyceraldehyde 3-phosphate dehydrogenase was applied for the internal normalization of RNA. The PCR reaction was performed at $95^{\circ} \mathrm{C}$ for $3 \mathrm{~min}$, followed by 40 cycles of $95^{\circ} \mathrm{C}$ for $10 \mathrm{~s}$ and $60^{\circ} \mathrm{C}$ for $30 \mathrm{~s}$. The comparative cycle threshold $(\mathrm{Ct})$ method $(\Delta \mathrm{Ct})$ was used to calculate the relative expression levels of miRs. The mean $\mathrm{Ct}$ values and deviations between the duplicates were calculated for all samples. Primers for the miR-30 were as follows: miR-30a, Forward CTAGCCTGCAGGATAAACTTACTCATGTTCTA3', Reverse ATCCGGCCGGCCTACTCTGAGATTTGATAAAT; miR-30b, Forward TAGCCTGCAGGTAGGTGGGAAAAGCTATAGA, Reverse ATCCGGCCGGCCGCTGACAAAGAAGTGGAGAC; miR-30c-1 Forward 5'-CTAGCCTGCAGGGACAGTTTGTCATGCAAGTA, reverse ATCCGGCCGGCCGCGATGGAAGATGCTACCCA; miR-30d, 5'-GATGATGACTGG CAACAT-3', and reverse: 5'-GAA TAG CCG GTA GCA GCA-3'; miR-30e Forward CTAGCCTGCAGGGGTTTAGTGTAATATGCCTC, Reverse ATCCGGCCGCCGCTCTTTAGTCATVATTTCA, $\beta$-actin, Forward AGAGCTACGAGCTGCCTGAC and reverse GGATGCCACAGGACTCCA ${ }^{16,23}$. For validation purposes, 20 samples were sent for sequencing and all PCR products were checked with the electrophoresis analyses.

\section{Statistical analysis}

The mean \pm standard deviation or median is presented as continuous variables with quartile ranges, and comparisons were conducted using a one-way ANOVA or the Mann-Whitney U-test. Categorical variables were described as numbers and percentages. The clinical parameters associated with $\mathrm{EH}$ risks were assessed by multivariable linear regression analysis. The relationships between the expressions of miR-30 family members and the LVMI were calculated using Pearson's correlation test. Then, the area under the receiver operating curve (ROC) was created to evaluate the potential value of miR-30. The area under the curve (AUC), sensitivity, and specificity were determined. Moreover, the logistic regression with backward elimination was performed to determine the independent predictors of the presence of $\mathrm{LVH}$. The crude odds ratio was adjusted after introducing factors including age, gender, smoking status, SBP, DBP, and DM, together with TC, TG, HDL$C$, LDL-C, as well as all the circulating levels of the miR-30 family members. All statistical analyses were performed with SPSS software 22.0 (IBM, Chicago, IL, USA) and GraphPad Prism 5.0 (GraphPad Software, La Jolla, CA, USA), and $p<0.05$ was considered statistically significant.

\section{RESULTS}

\section{Clinical features of patients and controls}

The baseline characteristics of all EH patients and healthy controls are detailed in Table 1. EH patients had significantly higher SBP, DBP as well as highsensitivity $\mathrm{C}$-reactive protein (hs-CRP) levels and a higher prevalence of DM. No differences in gender, age, smoking status, as well as BMI, TC, TG, LDL-C, and $\mathrm{HDL}-\mathrm{C}$ levels were found between the $\mathrm{EH}$ and control groups. The LV mass index (LVMI) was dramatically higher in EH patients than controls $(p<$ 0.001 ). As for circulating miR-30 levels, we found that circulating miR-30a, miR-30b, miR-30d, and miR-30e were significantly reduced in $\mathrm{EH}$ patients in contrast to controls (all $p<0.01$ ). The miR$30 c-1$ and miR-30c-2 levels were similar in the two groups. 
Table 1. Baseline characteristics of control and EH subjects

\begin{tabular}{|c|c|c|c|}
\hline Characteristic & Control (239) & $\mathrm{EH}(239)$ & $\mathrm{p}$-value \\
\hline Age & $63.6 \pm 5.5$ & $64.2 \pm 6.1$ & NS \\
\hline Male & $168(70.3 \%)$ & $172(72.0 \%)$ & NS \\
\hline BMI & $23.60 \pm 6.10$ & $24.10 \pm 7.5$ & NS \\
\hline SBP & $122.32 \pm 10.4$ & $151.64 \pm 13.4$ & $<0.001$ \\
\hline DBP & $66.24 \pm 7.52$ & $84.37 \pm 8.52$ & 0.016 \\
\hline Smokers & $80(33.50 \%)$ & $85(35.50 \%)$ & NS \\
\hline $\mathrm{TC}(\mathrm{mmol} / \mathrm{L})$ & $3.65 \pm 1.23$ & $3.72 \pm 1.12$ & NS \\
\hline $\mathrm{LDL}-\mathrm{C}(\mathrm{mmol} / \mathrm{L})$ & $1.97 \pm 0.61$ & $1.95 \pm 0.96$ & NS \\
\hline $\mathrm{HDL}-\mathrm{C}(\mathrm{mmol} / \mathrm{L})$ & $0.98 \pm 0.35$ & $0.97 \pm 0.52$ & NS \\
\hline $\mathrm{TG}(\mathrm{mmol} / \mathrm{L})$ & $1.48 \pm 0.57$ & $1.52 \pm 0.66$ & NS \\
\hline Diabetes & $56(23.43 \%)$ & $88(36.82 \%)$ & 0.024 \\
\hline Creatinine & $70.48 \pm 16.76$ & $76.66 \pm 18.67$ & NS \\
\hline Hs-CRP (mg/L) & $0.78 \pm 0.25$ & $1.54 \pm 0.68$ & 0.035 \\
\hline MMP-9 (ng/mL) & $338.9 \pm 78.6$ & $576.07 \pm 67.8$ & 0.013 \\
\hline LVMI & $88.5 \pm 13.7$ & $112.6 \pm 22.5$ & $<0.001$ \\
\hline MIR-30a & $9.85 \pm 2.05$ & $5.52 \pm 1.82$ & $<0.001$ \\
\hline$m i R-30 b$ & $8.65 \pm 1.54$ & $3.02 \pm 1.42$ & $<0.001$ \\
\hline$m i R-30 c-1$ & $4.65 \pm 1.21$ & $4.02 \pm 1.59$ & NS \\
\hline$m i R-30 c-2$ & $6.15 \pm 1.99$ & $5.82 \pm 1.18$ & NS \\
\hline miR -30d & $10.15 \pm 3.21$ & $7.02 \pm 2.36$ & 0.038 \\
\hline $\operatorname{miR}-30 e$ & $16.65 \pm 1.59$ & $8.12 \pm 1.16$ & $<0.001$ \\
\hline
\end{tabular}

BMI: body mass index; TC: total cholesterol; LDL-C: low-density lipoprotein cholesterol; HDL-C: high-density lipoprotein cholesterol; TG: triglycerides; Hs-CRP: high-sensitivity C-reactive protein; EH: essential hypertension; miR: microRNA; SBP: systolic blood pressure; DBP: diastolic blood pressure.

We subdivided the $\mathrm{EH}$ patients based on the presence (EH-LVH, $n=90$ ) or absence (EH-nLVH, $n=149$ ) of $\mathrm{LVH}$. As shown in Table 2, EH-LVH patients had substantially lower circulating miR-30b, miR-30d, and miR-30e levels compared to EH-nLVH patients ( $p=$ $0.017,0.011$, and $<0.001$, respectively). The other forms of miR-30 and baseline characteristics were similar between $\mathrm{EH}$ patients with and without $\mathrm{LVH}$.

We used Pearson's correlation test to evaluate the relationship between circulating miR-30 levels and baseline characteristics, such as age, SBP, DBP, hsCRP, TC, TG, HDL, LDL, and LVMI, of EH patients. We found that the expression level of miR-30b and miR30e was negatively correlated to LVMI $(r=-0.79$, $p$ $<0.001 ; r=-0.93, p<0.001)$, hs-CRP $(r=-0.74, p$ $<0.001 ; r=-0.88, p<0.001)$, and matrix metallopeptidase (MMP)-9 $(r=-0.69, p=0.022 ; r=-0.73$, $p=0.017)$, respectively.
The above data suggested that some miR-30 family members, especially the miR-30b and miR-30e, may serve as diagnostic markers for LVH in EH patients. We performed the ROC curve analysis to confirm this hypothesis. As shown in figure 1 (left), for miR-30e at a cutoff value of 3.74 , the AUC was $0.929(95 \% \mathrm{Cl}$, $0.785-0.976 ; \mathrm{p}<0.001$ ) with $88 \%$ sensitivity and $74 \%$ specificity to distinguish EH-LVH from EH-nLVH patients. Besides, as shown in figure 1 (right), the circulating miR-30e level is sufficient to differentiate $\mathrm{EH}$ from controls at a cutoff value of 3.68 (AUC was 0.843 , $95 \% \mathrm{Cl}, 0.804-0.896, \mathrm{p}<0.001$, with $79.1 \%$ sensitivity and $70.3 \%$ specificity). On the other hand, the miR$30 \mathrm{~b}$ failed to distinguish $\mathrm{EH}$ patients from controls and EH-LVH from EH-nLVH individuals (data not shown).

We then identified the risk factors for LVH using logistic regression analyses. In univariate analysis, the presence of DM, SBP $>160 \mathrm{mmHg}$, and circulating 
Table 2. Baseline characteristics of EH subjects with $(E H-L V H)$ and without (EH-nLVH) left ventricular hypertrophy

\begin{tabular}{|c|c|c|c|}
\hline Characteristic & EH-nLVH (149) & EH-LVH (90) & $\mathrm{p}$-value \\
\hline Age & $63.7 \pm 7.6$ & $65.4 \pm 5.3$ & NS \\
\hline Male & $108(72.48 \%)$ & $64(71.11 \%)$ & NS \\
\hline $\mathrm{BMI}$ & $23.70 \pm 5.18$ & $25.03 \pm 8.5$ & NS \\
\hline SBP & $152.54 \pm 9.88$ & $158.31 \pm 15.58$ & NS \\
\hline DBP & $84.54 \pm 6.67$ & $86.89 \pm 9.12$ & NS \\
\hline Smoking & $54(36.24 \%)$ & $31(24.44 \%)$ & NS \\
\hline $\mathrm{TC}(\mathrm{mmol} / \mathrm{L})$ & $3.68 \pm 1.05$ & $3.96 \pm 1.56$ & NS \\
\hline LDL (mmol/L) & $1.93 \pm 0.78$ & $2.02 \pm 1.02$ & NS \\
\hline $\mathrm{HDL}(\mathrm{mmol} / \mathrm{L})$ & $0.94 \pm 0.65$ & $1.03 \pm 0.98$ & NS \\
\hline $\mathrm{TG}(\mathrm{mmol} / \mathrm{L})$ & $1.48 \pm 0.96$ & $1.63 \pm 0.38$ & NS \\
\hline Diabetes & $57(38.26 \%)$ & $31(34.44 \%)$ & NS \\
\hline Creatinine & $74.41 \pm 19.11$ & $78.66 \pm 18$ & NS \\
\hline Hs-CRP (mg/L) & $1.45 \pm 0.63$ & $1.97 \pm 0.82$ & 0.024 \\
\hline MMP-9 (ng/mL) & $649.5 \pm 98.8$ & $527.07 \pm 47.8$ & 0.018 \\
\hline LVMI & $136.6 \pm 14.8$ & $87.6 \pm 1$ & $<0.001$ \\
\hline miR-30a & $5.78 \pm 1.28$ & $5.34 \pm 1.18$ & NS \\
\hline miR-30b & $3.57 \pm 1.57$ & $2.44 \pm 1.06$ & 0.017 \\
\hline miR-30c-1 & $4.15 \pm 1.27$ & $3.92 \pm 1.79$ & NS \\
\hline miR-30c-2 & $5.73 \pm 1.49$ & $6.02 \pm 1.48$ & NS \\
\hline miR-30d & $8.28 \pm 3.67$ & $5.24 \pm 2.28$ & 0.011 \\
\hline miR-30e & $10.87 \pm 1.95$ & $5.08 \pm 2.04$ & $<0.001$ \\
\hline
\end{tabular}

BMI: body mass index; TC: total cholesterol; LDL-C: low-density lipoprotein cholesterol; HDL-C: high-density lipoprotein cholesterol; TG: triglycerides; Hs-CRP: high-sensitivity C-reactive protein; MMP-9: matrix metallopeptidase 9; LVH: left ventricular hypertrophy; EH: essential hypertension; miR: microRNA; SBP: systolic blood pressure; DBP: diastolic blood pressure.

miR-30e $<3.74$ was each significantly associated with greater odds of having LVH (Table 3 ). In the multivariate analysis, SBP $>160 \mathrm{mmHg}$ or circulating miR30 e $<3.74$ was identified as independent risk factors for LVH in the study cohorts (Table 3 ).

\section{DISCUSSION}

In this study, we evaluated the clinical significance of circulating levels of miR-30 family members as clinical markers for $\mathrm{LVH}$ in $\mathrm{EH}$ patients. We found that most of miR-30 family members (except miR-30c1 and c2) were lower in $\mathrm{EH}$ patients with LVH compared to those without LVH. Further analyses revealed that miR-30e could efficiently discriminate $\mathrm{EH}-\mathrm{LVH}$ from $\mathrm{EH}-\mathrm{nLVH}$ patients. This finding confirms the role of miR-30e in a clinical setting and provides a novel biomarker for $\mathrm{LVH}$ in $\mathrm{EH}$ patients.
The miR-30 family members are encoded by six genes located on human chromosomes 1,6 , and $8^{10,13}$. The miR-30 family has different compensatory sequences near the $3^{\prime}$ end, allowing each member to target different genes and pathways, thus performing corresponding biological functions ${ }^{13}$. For example, miRNAs-30a-5p inhibits modulating GRP78 expression and suppresses the growth of renal cell carcinoma ${ }^{24}$. Mir-30d suppresses colon cancer cell growth by inhibiting cell genes regulating autophagy ${ }^{25}$. miRNA 30e regulates smooth muscle cells response to oxidative stress through Snail in an atherosclerosis animal model 26 .

To the best of our knowledge, this study is the first to report the clinical significance of miR-30e as a biomarker for $\mathrm{LVH}$ in $\mathrm{EH}$ patients. The role of miR-30e in cardiovascular diseases has been reported previously. For example, miR-30e in the myocardium is 
Figure 1. Graphs show ROC analyses of circulating microRNA-30e levels. A: In the analysis for distinguishing essential hypertension-left ventricular hypertrophy $(\mathrm{EH}-\mathrm{LVH})$ from $\mathrm{EH}-\mathrm{NLVH}$ patients, the AUC was $0.929(95 \% \mathrm{Cl}, 0.785-0.976 ; \mathrm{p}<0.001)$ with $88 \%$ sensitivity and $74 \%$ specificity (cutoff value, 3.74 ). B: In distinguishing EH patients from healthy controls, the AUC was 0.843 ( $95 \% \mathrm{Cl}, 0.804-0.896, p<0.001$ ), with $79.1 \%$ sensitivity and $70.3 \%$ specificity (cutoff value, 3.68 ). $p<0.05$ was considered statistically significant. ROC: receiver operating curve, AUC: area under the curve.

A

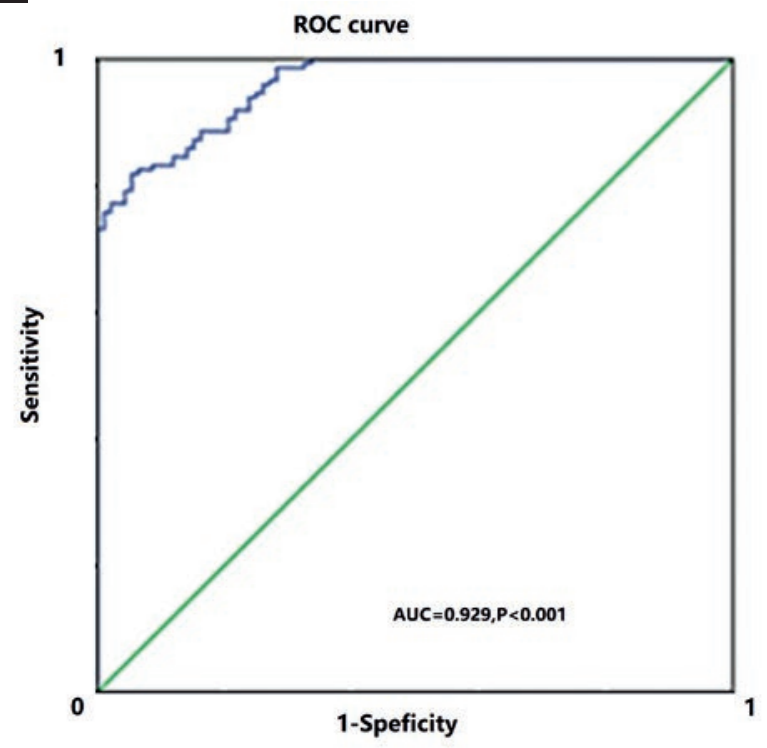

B

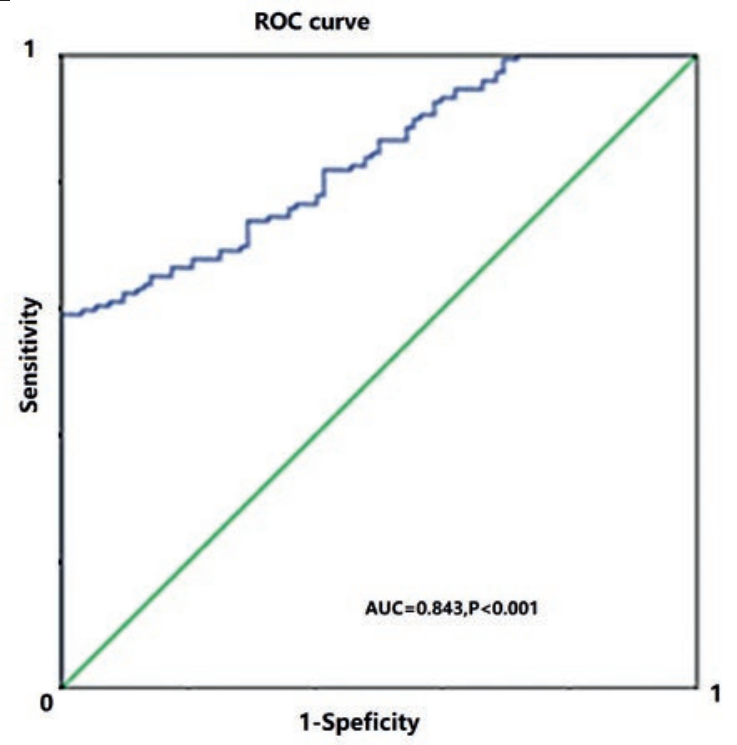

Table 3. Risk factors for LVH identified by logistic regression analyses

\begin{tabular}{|c|c|c|c|c|c|c|}
\hline \multirow[t]{2}{*}{ Variables } & \multirow{2}{*}{$\begin{array}{c}\text { Hazard } \\
\text { ratio }\end{array}$} & \multirow{2}{*}{$\begin{array}{c}\text { Univariate } \\
95 \% \mathrm{Cl}\end{array}$} & \multirow{2}{*}{$\begin{array}{c}\text { Analysis } \\
p \text {-value }\end{array}$} & \multirow{2}{*}{$\begin{array}{c}\text { Hazard } \\
\text { ratio }\end{array}$} & \multirow{2}{*}{$\begin{array}{c}\text { Multivariate } \\
95 \% \mathrm{Cl}\end{array}$} & \multirow{2}{*}{$\begin{array}{c}\text { Analysis } \\
\text { p-value }\end{array}$} \\
\hline & & & & & & \\
\hline \multicolumn{7}{|l|}{ DM } \\
\hline No & 1 & & & 1 & & \\
\hline Yes & 1.36 & $1.08-2.54$ & 0.036 & 1.18 & $0.95-2.47$ & 0.056 \\
\hline \multicolumn{7}{|c|}{$\mathrm{SBP}>160 \mathrm{mmHg}$} \\
\hline No & 1 & & & 1 & & \\
\hline Yes & 1.88 & $1.23-4.02$ & 0.008 & 1.94 & $1.28-4.17$ & 0.006 \\
\hline \multicolumn{7}{|l|}{ miR-30e } \\
\hline$>3.74$ & 1 & & & 1 & & \\
\hline$<3.74$ & 2.25 & $1.47-6.45$ & 0.004 & 2.03 & $1.66-6.02$ & 0.003 \\
\hline
\end{tabular}

LVH: left ventricular hypertrophy; miR: microRNA; SBP: systolic blood pressure; DM: diabetes mellitus.

inhibited after the occurrence of coronary microembolization, together with decreased cardiac function $^{27}$. miR-30e alleviates apoptosis induced by hypoxia in human stem cell-derived cardiomyocytes ${ }^{28}$. Of note, the effect of miR-30e on cardiomyocyte hypertrophy was reported in a series of cellular and animal studies. MiR-30e can repress the Angiotensin Il-induced hypertrophic phenotypes in cardiomyocytes by targeting ADAM929. Yin et al. found that myocardial expression of miR-30a was decreased in mouse models of hypertrophy, and in H9c2 cells treated with phenylephrine by activating autophagy 21 . It is reported that this miR can reduce isoproterenolinduced cardiac dysfunction and fibrosis in a rat cardiac remodeling model ${ }^{30}$. The authors of this study observed a significant reduction of miR-30e in the cardiac samples of isoproterenol-treated rats alone with cardiac fibrosis. In contrast, the in vivo 
administration of miR-30e antagomir increased the survival of ISO-treated rats.

Other miR30 members, such as miR-30a, have also been reported to alleviate cardiac fibrosis in rats that underwent myocardial infarction ${ }^{31}$. The overexpression of miR-30c reduces cardiac fibrosis, and inhibition of miR-30 leads to collagen synthesis in heart tissue ${ }^{18}$. However, our study did not prove its clinical value in patients.

Mechanistically, the miR-30 family can inhibit autophagy and apoptosis, decrease inflammation, etc., thereby reducing collagen deposition and myocardial fibrosis, and preventing cardiac hypertrophy ${ }^{19}$. We thus detected the correlation between miR-30 family members and the circulating markers for inflammation (hs-CRP) and collagen synthesis (MMP-9). We found that only miR-30e showed a negative correlation with serum hs-CRP and MMP-9, suggesting that the patients with lower miR-30e have a relatively low level of inflammation and cardiac fibrosis.

A major limitation should be addressed in this study. Our current study is a single-center based clinical research with a small sample size. To validate the value of circulating miR-30e in LVH detection, a multi-centered prospective study with a larger sample size is warranted.

\section{REFERENCES}

1. Ajayi EA, Ajayi IA, Oyedeji TA, Adeoti AO, Omotoye OJ, Adebayo RA. Left ventricular mass formulae and prevalence rates of echocardiographic left ventricular hypertrophy in Nigerians with essential hypertension. N Am J Med Sci. 2013;5:325-9.

2. Palmiero P, Maiello M. Ventricular arrhythmias and left ventricular hypertrophy in essential hypertension. Minerva Cardioangiol. 2000;48:427-34

3. Yucel C, Demir S, Demir M, Nas K, Molnar F, Illyes M, et al. Left ventricular hypertrophy and arterial stiffness in essential hypertension. Bratisl Lek Listy. 2015;116:714-8.

4. Tovillas-Moran FJ, Vilaplana-Cosculluela M, Zabaleta-del-Olmo E, Dalfo-Baque A, Galceran JM, Coca A. Cardiovascular morbidity and mortality and electrocardiographic criteria of left ventricular hypertrophy in hypertensive patients treated in primary care. Med Clin (Barc). 2010;135:397-401.

5. Sharpe N. Correlations of left ventricular hypertrophy with cardiovascular mortality. Int J Clin Pract Suppl. 2000;110:16-22.

6. Cai Y, Yu X, Hu S, Yu J. A brief review on the mechanisms of miRNA regulation. Genomics Proteomics Bioinformatics. 2009; 7:147-54.

7. Jiang L, Zhu J. Review of MiRNA-disease association prediction. Curr Protein Pept Sci. 2020; [Epub ahead of print]

8. Powrozek T, Malecka-Massalska T. MiRNA and lung cancer radiosensitivity: a mini-review. Eur Rev Med Pharmacol Sci. 2019;23:8422-8
9. Wang T, Xu H, Qi M, Yan S, Tian X. miRNA dysregulation and the risk of metastasis and invasion in papillary thyroid cancer: a systematic review and meta-analysis. Oncotarget. 2018; 9:5473-9.

10. Feng B, Chen S, George B, Feng Q, Chakrabarti S. miR133a regulates cardiomyocyte hypertrophy in diabetes. Diabetes Metab Res Rev. 2010;26:40-9.

11. Ucar A, Gupta SK, Fiedler J, Erikci E, Kardasinski M, Batkai S, et al. The miRNA-212/132 family regulates both cardiac hypertrophy and cardiomyocyte autophagy. Nat Commun. 2012; 3:1078.

12. Yang T, Liu T, Cao C, Xu S. miR-200a-5p augments cardiomyocyte hypertrophy induced by glucose metabolism disorder via the regulation of selenoproteins. J Cell Physiol. 2019;234: 4095-103.

13. Goretti E, Zangrando J, Wagner DR, Devaux Y. Unity is strengtha panel of 4 microRNAs decreases cardiomyocyte hypertrophy. Int J Cardiol. 2015;182:62-4.

14. Lai L, Chen J, Wang N, Zhu G, Duan X, Ling F. MiRNA-30e mediated cardioprotection of ACE2 in rats with doxorubicin-induced heart failure through inhibiting cardiomyocytes autophagy. Life Sci. 2017;169:69-75.

15. Li Y, Maegdefessel L. My heart will go on-beneficial effects of anti-MiR-30 after myocardial infarction. Ann Transl Med. 2016; $4: 144$.

16. Mao L, Liu S, Hu L, Erikci E, Kardasinski M, Batkai S, et al. miR-30 family: a promising regulator in development and disease. Biomed Res Int. 2018:2018:9623412.

17.Pan W, Zhong Y, Cheng C, Liu B, Wang L, Li A, et al. MiR-30-regulated autophagy mediates angiotensin II-induced myocardial hypertrophy. PLoS One. 2013;8:e53950.

18. Duisters RF, Tijsen AJ, Schroen B, Leenders JJ, Lentink V, van der Made I, et al. miR-133 and miR-30 regulate connective tissue growth factor: implications for a role of microRNAs in myocardial matrix remodeling. Circ Res. 2009;104:170-8.

19. Zhang X, Dong S, Jia Q, Zhang A, Li Y, Zhu Y, et al. The microRNA in ventricular remodeling: the miR-30 family. Biosci Rep. 2019;39:0788

20. Duan Q, Chen C, Yang L, Li N, Gong W, Li S, et al. MicroRNA regulation of unfolded protein response transcription factor XBP1 in the progression of cardiac hypertrophy and heart failure in vivo. J Transl Med. 2015;13:363

21. Yin X, Peng C, Ning W, Li C, Ren Z, Zhang J, et al. miR-30a downregulation aggravates pressure overload-induced cardiomyocyte hypertrophy. Mol Cell Biochem. 2013;379:1-6.

22. Wang Y, Chen S, Gao Y, Zhang S. Serum MicroRNA-27b as a screening biomarker for left ventricular hypertrophy. Tex Heart Inst J. 2017;44:385-9.

23. Zhou Y, Hao Y, Li Y, Li R, Wu R, Wang S, et al Amplification and up-regulation of MIR30D was associated with disease progression of cervical squamous cell carcinomas. BMC Cancer. 2017; $17: 230$.

24. Wang C, Cai L, Liu J, Wang G, Li H, Wang X, et al. MicroRNA-30a$5 p$ inhibits the growth of renal cell carcinoma by modulating GRP78 expression. Cell Physiol Biochem. 2017;43:2405-19.

25. Zhang R, Xu J, Zhao J, Bai J. Mir-30d suppresses cell proliferation of colon cancer cells by inhibiting cell autophagy and promoting cell apoptosis. Tumour Biol. 2017;39 [Epub ahead of print].

26. Cheng Y, Zhou M, Zhou W. MicroRNA-30e regulates TGF-betamediated NADPH oxidase 4-dependent oxidative stress by Snai1 in atherosclerosis. Int J Mol Med. 2019;43:1806-16.

27. Wang $X T$, Wu XD, Lu YX, Sun $Y$, Zhu H, Liang J, et al. Potential Involvement of MiR-30e-3p in myocardial injury induced by coronary microembolization via autophagy activation. Cell Physiol Biochem. 2017;44:1995-2004.

28. Mo B, Wu X, Wang X, Xie J, Ye Z, Li L. miR-30e-5p mitigates hypoxia-induced apoptosis in human stem cell-derived cardiomyocytes by suppressing bim. Int J Biol Sci. 2019;15:1042-51.

29. Wang W, Wu C, Ren L, Bao Y, Han Y, Li C, et al. MiR-30e-5p is sponged by Kcnq1ot 1 and represses angiotensin Il-induced hypertrophic phenotypes in cardiomyocytes by targeting ADAM9. Exp Cell Res. 2020;2020:112140

30. Zhang W, Chang H, Zhang H, Zhang L. MiR-30e attenuates isoproterenol-induced cardiac fibrosis through suppressing Snai1/TGF-beta signaling. J Cardiovasc Pharmacol. 2017; 70:362-8

31. Maciejak A, Kostarska-Srokosz E, Gierlak W, Dluzniewski M, Kuch M, Marchel M, et al. Circulating miR-30a-5p as a prognostic biomarker of left ventricular dysfunction after acute myocardial infarction. Sci Rep. 2018;8:9883. 